\title{
Compact Directive On-body UWB Antenna for Wireless Capsule Endoscopy Systems
}

\author{
Chaïmaâ Kissi \\ Electronic Electronics and \\ Telecommunication Systems Research \\ Group, National School of Applied \\ Sciences (ENSA) \\ Ibn Tofail University \\ Kenitra, Morocco \\ chaimaakissi1@gmail.com \\ Sami Myllymäki \\ Microelectronics Research Unit, \\ Faculty of Information Technology and \\ Electrical Engineering \\ University of Oulu \\ Oulu, Finland \\ sami.myllymaki@oulu.fi
}

Heli Jantunen

Microelectronics Research Unit,

Faculty of Information Technology and

Electrical Engineering

University of Oulu

Oulu, Finland

heli.jantunen@oulu.fi

Abstract-Wireless Capsule Endoscopy (WCE) application is a challenging topic inciting a good designed receiving antenna, in order to improve the wireless communication with the capsule endoscope. In this context, the paper introduces a compact antenna operating at the low Ultra-Wide ban d (UWB) 3.75-4.25 GHz, defined in IEEE 802.15.6 standard for BAN (Body Area Networks) applications. The proposed UWB antenna is targeted to on-body use, to serve of receiving antenna, and has a total size of $30 \times 25 \times 1.6 \mathrm{~mm} 3$. In free space, the antenna has a directional radiation pattern with $9 \mathrm{~dB}$ measured gain at $4 \mathrm{GHz}$ center frequency, and it covers the $10 \mathrm{~dB}$ impedance ban dwidth of 3.75-4.45 GHz range. Then, the antenna was placed at different distances next to a voxel model, to consider the human body effect on the antenna characteristics. Besides, measurements were carried out on a male person by taking into account two antenna positions, named "Parallel" and "perpendicular", on the human body surface. The proposed antenna does not present any risk on the person safety. The maximum $S A R$ value is estimated of $0.095 \mathrm{~W} / \mathrm{kg}$ for the different positions, in compliance with IEEE C95.3 standard on $10 \mathrm{~g}$ average tissue. The on-body matching studies were followed by in-body investigations, by analyzing the power consumption by means of power-field probes, from skin to the Small Intestine (SI) layers in voxel model. All the conducted studies conclude that the antenna, in both positions, is a good receiving antenna suitable for Wireless Capsule Endoscopy communications.

Keywords-Antenna position, Directive radiation pattern, high gain, IEEE 802.15.6 standard, SAR, small-intestine, WBAN.

\section{INTRODUCTION}

In recent years, many researches have been launched for medical applications, like wire less capsule endoscopy. This

\author{
Timo Kumpuniemi \\ Centre for Wireless Communications, \\ Faculty of Information Technology and \\ Electrical Engineering \\ University of Oulu \\ Oulu, Finland \\ timo.kumpuniemi@oulu.fi
}

\author{
Mohamed Nabil Srifi \\ Electronic Electronics and \\ Telecommunication Systems Research \\ Group, National School of Applied \\ Sciences (ENSA) \\ Ibn Tofail University \\ Kenitra, Morocco \\ srifimn@gmail.com
}


$100 \mathrm{~mm}$-length. This on-body antenna has a relatively small size compared with the horn antenna, but its measured $10 \mathrm{~dB}$ covered bandwidth is very poor in the sense that the maximum reflection coefficient does not exceed $-12 \mathrm{~dB}$ which constraints the good operation of the antenna for this kind of medical applications. In [10] a trapezo id monopole receiving antenna is proposed to establish the data telemetry lin $\mathrm{k}$ with the designed capsule. In fact, this on-body antenna has proven to efficiently operate in $3-5 \mathrm{~dB}$ frequency range with a measured gain of $5.8 \mathrm{~dB}$ at $4 \mathrm{GHz}$. It is clearly seen that available wireless capsule endoscopy papers give emphasize on the capsule antenna by leaving besides the well-design of the receiving antennas. However, it is prevalent to design a receiving antenna with good directivity towards the human body surface and predict the human body effect with the separation distance from the antenna. For this purpose, the paper aims to provide a novel on-body receiving antenna properly designed for in-body communication, with on/in-body investigations and concise measurements analysis. Consequently, the proposed antenna structure has higher antenna performances in terms of covered bandwidth, resonant frequency and maximum achieved gain compared with the reported ones in literature.

This paper comes to provide and take advantage of a simulation and measurement-based on-body study for an UWB receiving on-body antenna suitable for wireless capsule endoscopy systems. The paper defines a compact antenna structure operating over $3.75-4.25 \mathrm{GHz}$ UWB band of IEEE 802.15.6 standard [11]. The antenna replies to the main features required by a good on-body receiving antenna, which is the good directivity towards body surface. Initially, the antenna was developed in free-space, as described in Section 2. Then, on-body performance of the antenna was analyzed and discussed in Section 3. Finally, the simulated in-body antenna performance was investigated in Section 4. It is concluded that the proposed antenna represent a good antenna element for the establishment of a communication link with low path loss between a capsule intended to be placed at the SI (Small-Intestine) tract and the proposed on-body receiving antenna.

\section{ANTENNA DESIGN AND PROTOTYPE}

The planar antenna was originally presented in [12] by applying a cavity-backed approach to the planar antenna configuration, as can be found in [13-14]. However, since no analysis was conducted previously using the presented antenna in its planar structure, this paper comes to provide a concise investigation with the compact planar antenna shown in Fig. 1, with detailed parameter values listed in [12]. The simulated model of the developed compact antenna is presented in Fig. 1 (a). We recall that the antenna is a dipole type and is printed on FR4 laminate of $1.6 \mathrm{~mm}$ thickness, $25 \mathrm{~mm}$ length and $30.3 \mathrm{~mm}$ width. Its radiating element has an inverted L-shape, while the ground plane is a composition of rectangular and inverted L-shapes. For more details, an accurate parametric study was presented and discussed in [12]. The manufactured antenna is illustrated in Fig. 1 (b) from front and back sides. An L-connector was used to connect the antenna to the coaxial cable, as figured in Fig. 1 (c), corresponding to the coordinate system presented in Fig. 1 (a). The antenna was measured according to the setup described in Fig. 1(d). The antenna performances in terms of reflection coefficient and radiation properties were measured to validate the simulated results. Agilent 8720A Vector Network Analyzer and Satimo StarLab, Fig. 1 (d), were used in the measurements.

The comparison of simulated and measured reflection coefficient, in free-space, of the proposed UWB antenna is presented in Fig. 2(a). Assuming that the reflection coefficient is defined below $-10 \mathrm{~dB}$, it is clearly seen that the lower frequency remains $3.75 \mathrm{GHz}$ while the upper frequency was raised by $170 \mathrm{MHz}$. Therefore, the UWB antenna has a measured impedance bandwidth of 3.754.45 GHz. Reflection coefficient results in Fig. 2 (a) prove a good agreement between measurements and simulations. Furthermore, the measured total efficiency in free-space presented in Fig. 2(b) confirms the good radiation properties over the considered $3.75-4.25 \mathrm{GHz}$ bandwidth, essentially at $4 \mathrm{GHz}$ center frequency with $-0.25 \mathrm{~dB}$ total efficiency corresponding roughly to $90 \%$ of the power received is radiated by the antenna.

The free-space antenna radiation features were performed in Fig. 3 at different cutting plans $\mathrm{Phi}=0^{\circ}, \mathrm{Phi}=90^{\circ}$ and Theta $=90^{\circ}$. The resulting radiation patterns were measured and compared to the simulated ones at $3.75 \mathrm{GHz}, 4 \mathrm{GHz}$ and 4.25 GHz as presented in Fig. 3 (a), Fig. 3 (b) and Fig. 3 (c), respectively. Globally, the shape of the radiation patterns follows the simulated ones according to the three cutting plans, at $3.75 \mathrm{GHz}, 4 \mathrm{GHz}$ and $4.25 \mathrm{GHz}$. The proposed antenna is a planar dipole type, which means that the antenna is a balanced structure fed, in this work, by a coaxial cable known as unbalanced feeding means. This only, justifies the fluctuations seen in the pattern plots at $\mathrm{Phi}=0^{\circ}$ and $\mathrm{Phi}=90^{\circ}$. Apart from that, at Theta $=90^{\circ}$, the pace of the measured radiation pattern quite overlap with the simulated one. According to Fig. 3, simulations predict a stable gain, in free-space, reaching a maximum gain value of $9 \mathrm{~dB}$ over the $3.75-4.25 \mathrm{GHz}$ operating bandwidth. Besides, according to Fig. 3, the proposed UWB antenna has a directional radiation pattern directed mainly towards $\mathrm{Y}$ axis. From the practical point of view, the proposed UWB antenna is a good candidate for a directive receiving antenna operating in free-space.

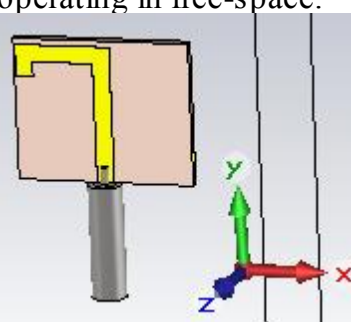

(a)

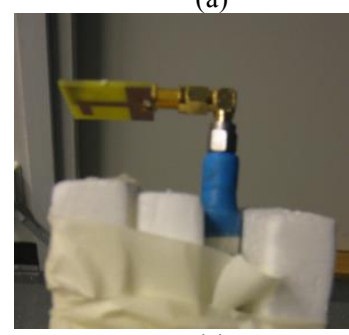

(c)

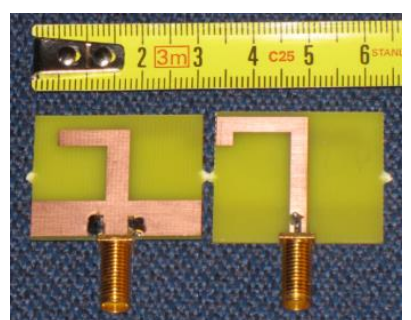

(b)

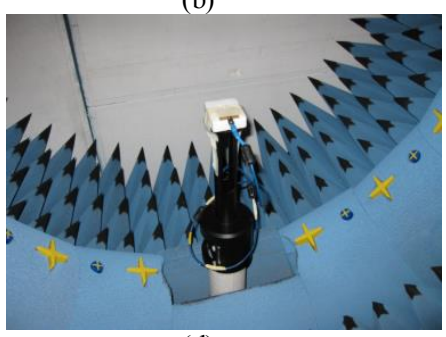

(d)
Fig. 1. (a) Simulated antenna model (b) Antenna prototype (c) antenna holding mechanism and (d) a setup for radiation pattern measurements. 


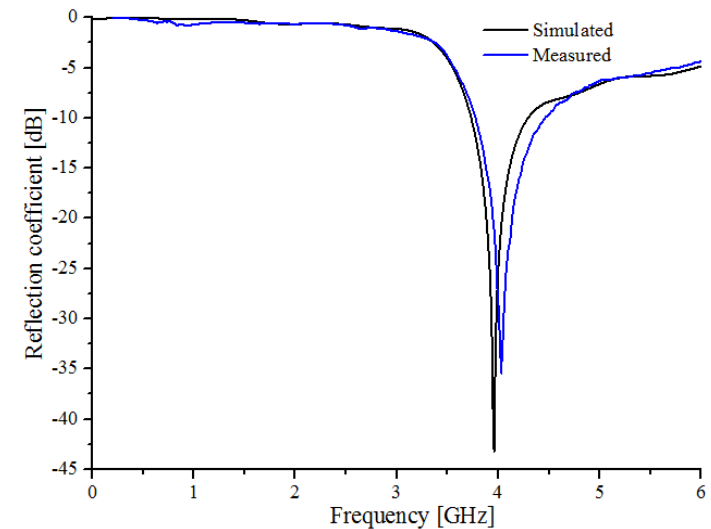

(a)

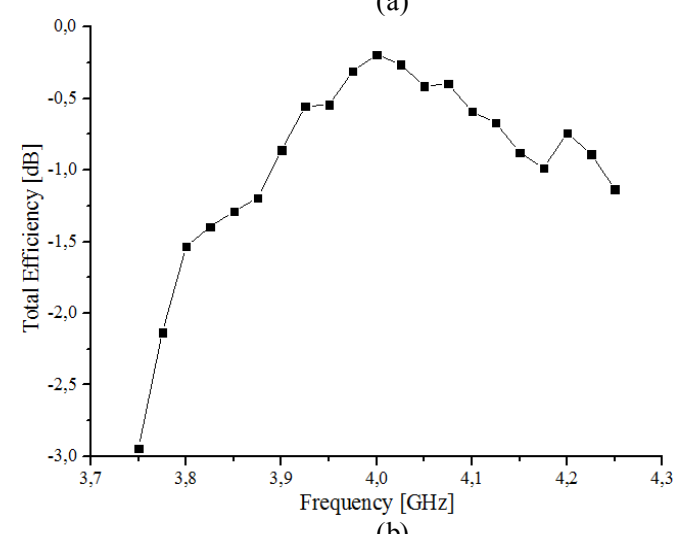

(b)

Fig. 2. (a) Simulated and measured reflection coefficient of the proposed UWB antenna, in free-space and (b) Measured total efficiency of the proposed UWB antenna, in free-space.

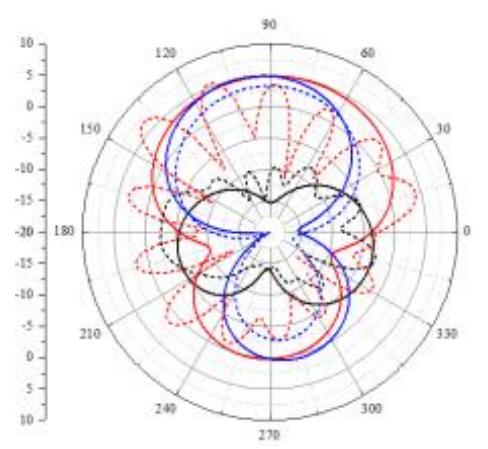

(a)

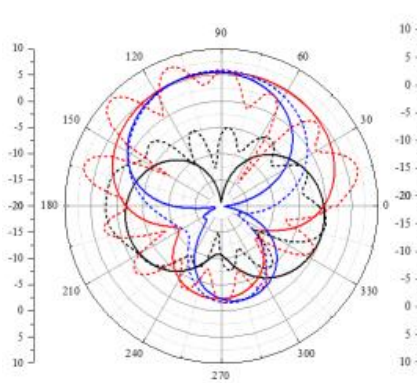

(b)

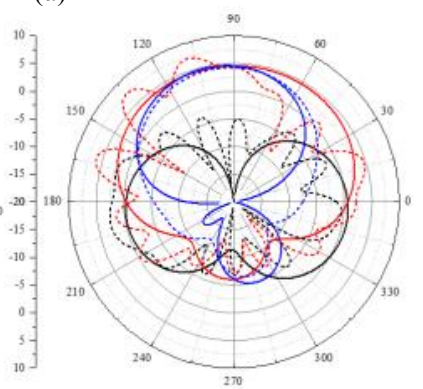

(c)
Fig. 3. Simulated and measured radiation patterns in $\mathrm{dB}$ of the proposed UWB antenna at (a) $3.75 \mathrm{GHz}$ (b) $4 \mathrm{GHz}$ and (c) $4.25 \mathrm{GHz}$.

\section{ON-BODY INVEST IGATIONS}

In this section, a detailed on-body study is presented using the proposed compact antenna. The simulated and measured results are compared and discussed.

\section{A. Simulated results}

To simulate the on-body use of the proposed antenna, a voxel model was used to realistically emulate the human body (Small Intestine area), as presented in Fig. 4. This subsection consists of the assessment of the antenna in two different positions with different separation distances $d$ (antenna-skin space), as mentioned in Fig. 4. The distance was limited to $4 \mathrm{~mm}$ and $30 \mathrm{~mm}$ in this paper. Later other separation distances will be tested. Assuming $\mathrm{d}=4 \mathrm{~mm}$ is averaging the clothes thickness for a realistic measurement case involving volunteers. The two agreed antenna positions under test in this paper are: "Perpendicular" to the abdomen area, where antenna is placed vertically to the skin surface, and "Parallel" to the abdomen area, where antenna is placed horizontally to the skin surface, as illustrated in Fig. 4 (a) and Fig. 4 (b).

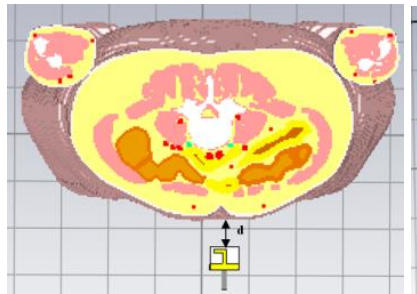

(a)

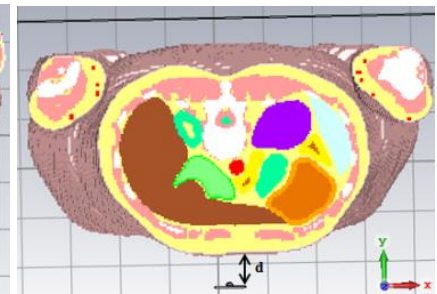

(b)
Fig. 4. UWB antenna next to the voxel model separated by distanced from the voxel skin surface in (a) "Perpendicular" and (b) "Parallel" positions.

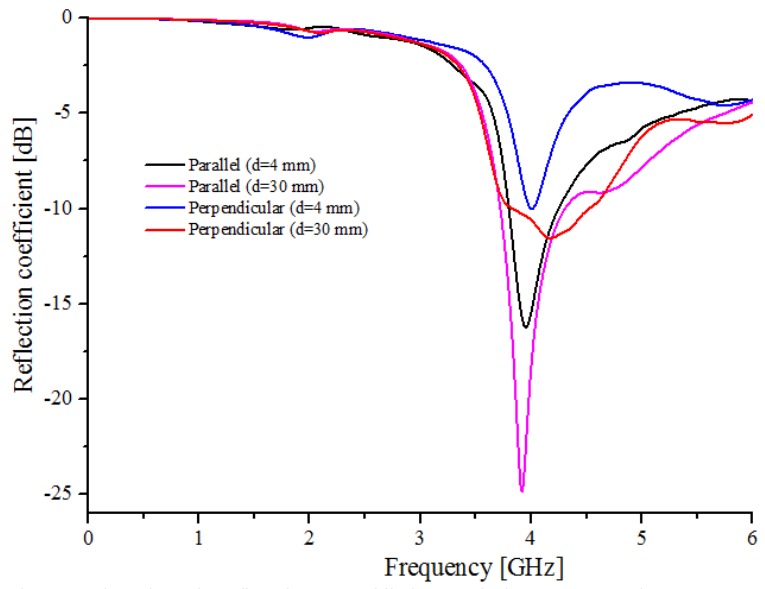

Fig. 5. Simulated reflection coefficient of the proposed UWB antenna in "Parallel" and "Perpendicular" position with different distances of $4 \mathrm{~mm}$ and $30 \mathrm{~mm}$ from the voxel model surface.

The body effect on the antenna matching characteristics was also evaluated on the selected female voxel model, recognized as Laura model 2018, from CST Library. The choice of this voxel model was emphasized on the inclusion/availability of the entire layer organs in need for this work, such as skin, visceral and subcutaneous fat, SI (Small-Intestine) wall and SI content layers to be close to the realistic case involving real candidates. Only a segment of the model was considered for this study to minimize the simulation time. The body part under focus was the navel area of the belly part, as illustrated in Fig. 4, since this position guarantees an efficient location aiming to reach an important amount of the meandered SI tract. The boundary conditions were fixed to "Open" state below and above the voxel segment in order to avoid any overflow of the antenna radiation features. Meanwhile the state was set to "Open (Add space)" in the front and back of the body model with the aim to meet realistic measurement conditions. The matching analysis of the proposed UWB antenna was restricted to the evaluation of the reflection coefficient result 
using the ofermentionned antenna positions "Parallel" and "Perpendicular", as presented in Fig. 5. It is worth noting that the parallel position achieved a $-10 \mathrm{~dB}$ impedance bandwidth close to the free-space case. In this context, one can notice that at $d=30 \mathrm{~mm}$, the reported bandwidth is $3.73-4.30 \mathrm{GHz}$ which is very close to meet the free-space one of 3.73-4.28 GHz. By applying a further distance decrease to $d=4 \mathrm{~mm}$, the bandwidth was slighlty narrowed to achieve 3.80-4.25 GHz. For "Perpendicular" antenna position, the reflection coefficient plots were dramatically deteriorated at both distances $d$. At this regard, for $d=30 \mathrm{~mm}$, a very poor $-10 \mathrm{~dB}$ impedance matching was seen as seen in Fig. 5. While by further getting closer to the body surface, e.g. $4 \mathrm{~mm}$, the bandwidth is getting worst until to not be covered. Then, it is worth noting that the bandwidth matching is satisfactory at $d=30 \mathrm{~mm}$ and especially when the antenna position is parallel. This issue will be confirmed by on-body measurements in the next section.

Before executing on-body measurements, the Specific Absorption Rate (SAR) value is worth of being estimated to ensure that the antenna does not cause any harmful effects on the pers on under test. In this context, SAR calculations were simulated in CST calculations for "Parallel" and "Perpendicular" positions with $4 \mathrm{~mm}$ separation distance. The calculation was carried out, in compliance with IEEE C95.3 standard [15], on a tissue volume of $2.62 \mathrm{E} 6 \mathrm{~mm} 3$ with an input power around $0.00316 \mathrm{~W}$. The reported total SAR values are $0.62 \mathrm{E}-3$ and $0.74 \mathrm{E}-3 \mathrm{~W} / \mathrm{Kg}$ in "Parallel" and "Perpendicular" positions. The maximum SAR value, by averaging $10 \mathrm{~g}$ tissue, is $0.095 \mathrm{~W} / \mathrm{kg}$ for "Parallel" and "Perpendicular" positions, respectively. Those values are far below the SAR limit authorized by common safety standards.

\section{B. Measurement results}

The performance of the proposed compact UWB antenna, in terms of the impedance matching, was measured in close proximity to real human body. The candidate under test was a male person wearing a thin t-shirt. The antenna was placed on the belly, in particular on the Navel, with different antenna-clothes separation distance $\mathrm{d}$ of $0 \mathrm{~mm}$, $2 \mathrm{~mm}, 4 \mathrm{~mm}, 6 \mathrm{~mm}, 8 \mathrm{~mm}, 10 \mathrm{~mm}, 20 \mathrm{~mm}$ and $30 \mathrm{~mm}$, as illustrated in Fig. 6 (a). The separation d was obtained by Rohacell pieces of different thicknesses. The reflection coefficient was measured by using Agilent 8720A Vector Network Analyzed. Measurements were conducted in an office room at Oulu University, Fin land. The two antenna positions named "Perpendicular" and "Parallel" were tested, and a $90^{\circ}$ bended coaxial connector was used to obtain the first position, as depicted in Fig. 6 (b). Comparison results are provided by Fig. 7 (a) and Fig. 7 (b), and detailed in Table 1, for "Perpendicular" and "Parallel" antenna positions, respectively. These measured results will be analyzed and discussed separately per antenna position. For "Perpendicular" position, the measured $-10 \mathrm{~dB}$ impedance bandwidth in free-space is $3.75-4.50 \mathrm{GHz}$. By placing the antenna at $30 \mathrm{~mm}$ from the candidate clothes, the $-10 \mathrm{~dB}$ impedance bandwidth shifted downwards to reach 3.70 $4.27 \mathrm{GHz}$. By further decreasing the separation distance d, fro $\mathrm{m} 20 \mathrm{~mm}$ to $4 \mathrm{~mm}$, the $-10 \mathrm{~dB}$ impedance bandwidth was not obtained. For $2 \mathrm{~mm}$ separation distance, the antenna bandwidth below $-10 \mathrm{~dB}$ started to appear interestingly. However, this time this -10 impedance bandwidth was shifted upwards averaging 4.00-4.85 GHz for $\mathrm{d}=2 \mathrm{~mm}$ and $\mathrm{d}=0 \mathrm{~mm}$.
According to Table 1, by analyzing the "Parallel" position, the measured $-10 \mathrm{~dB}$ impedance bandwidth in freespace is $3.75-4.50 \mathrm{GHz}$, by directly connecting the antenna to the coaxial cable Huber+Suhner SUCOFLEX 104PEA, length $8 \mathrm{~mm}$. By placing the antenna in parallel to the candidate navel, with distances $\mathrm{d}$ from $30 \mathrm{~mm}$ to $10 \mathrm{~mm}$ by $10 \mathrm{~mm}$ steps, it is clearly seen that starting with $\mathrm{d}=30 \mathrm{~mm}$, the $-10 \mathrm{~dB}$ impedance bandwidth was shifted to the left to cover 3.70-4.43 GHz. Later, the distance d was softly decreased with $2 \mathrm{~mm}$ step. By decreasing the distance from $10 \mathrm{~mm}$ to $8 \mathrm{~mm}$, the $-10 \mathrm{~dB}$ impedance bandwidth was widened to cover $3.80-4.42 \mathrm{GHz}$ and remained stable at $\mathrm{d}=6 \mathrm{~mm}$. With $4 \mathrm{~mm}$ and $2 \mathrm{~mm}$ separation distances, no $10 \mathrm{~dB}$ impedance bandwidth was covered. Whereas, this impedance bandwidth starts to appear, with $\mathrm{d}=0 \mathrm{~mm}$, of $3.27-4.27$. It is clearly seen that when the antenna is directly placed on the real person by means of clothes, the required bandwidth of $3.75-4.25 \mathrm{GHz}$, originally defined as lowest channel of UWB band in IEEE 802.15.6 standard, is entirely covered. Back to voxel model matching results, one can clearly see that the good impedance matching at $\mathrm{d}=30 \mathrm{~mm}$ using "Parallel" antenna position is confirmed by measurements. Consequently, all the studied issues are in good agreement with voxel model results and hence this leads to promote the "Parallel" position for the requested requirements. This specific antenna position is more suitable to on-body practical use involving patients, in the sense that holding the antenna in this way will allow the movement comfort to the person. However, an exhaustive analys is will be conducted in later paragraphs to complete these investigations, appealing to power-field probes to draw conclusions with regard to in-body performances.

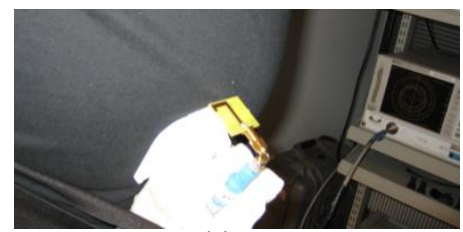

(a)

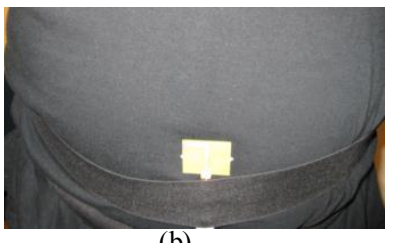

(b)
Fig. 6. On-body measurement setup in (a) "Perpendicular" and (b) "Parallel" positions.

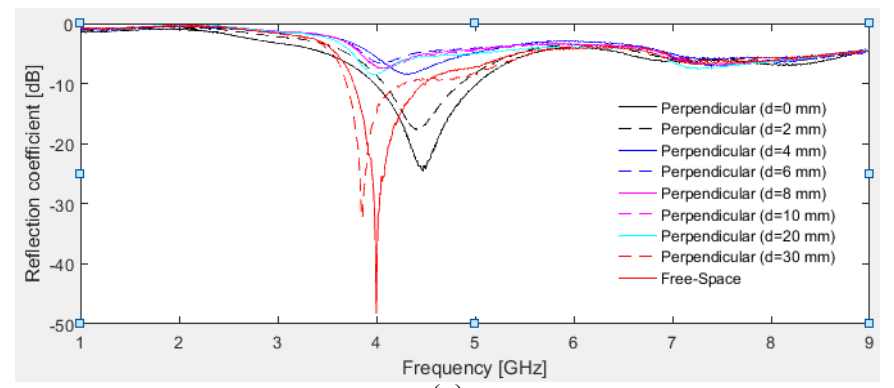

(a)

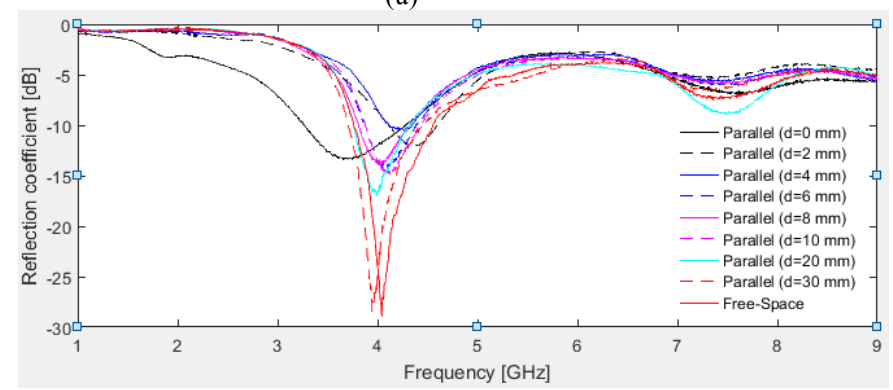

(b)

Fig. 7. Measured reflection coefficient of the proposed UWB antenna in (a) Perpendicular and (b) Parallel positions in close proximity to real human body. 
TABLE I: Measured reflection coefficient details of the proposed UWB antenna in perpendicular and parallel positions in vicinity to the human body

\begin{tabular}{|l|l|l|l|}
\hline & \multicolumn{3}{|c|}{ Perpendicular / Parallel Position } \\
\hline Perpendicular & Fr [GHz] & $\begin{array}{l}\text { Max.S11 } \\
{[\mathrm{dB}]}\end{array}$ & $\begin{array}{l}\text { Bandwidth } \\
{[\mathrm{dB}]}\end{array}$ \\
\hline$d=0 \mathrm{~mm}$ & $4.49 / 3.68$ & $\begin{array}{l}-23.94 /- \\
13.27\end{array}$ & $\begin{array}{l}3.98-4.85 \\
3.27-4.27\end{array}$ \\
\hline$d=2 \mathrm{~mm}$ & $4.43 / 4.37$ & $\begin{array}{l}-17.53 /- \\
11.98\end{array}$ & $\begin{array}{l}4.00-4.85 \quad / \\
*\end{array}$ \\
\hline$d=4 \mathrm{~mm}$ & $* / 4.22$ & $* /-10.29$ & $* / *$ \\
\hline$d=6 \mathrm{~mm}$ & $* / 4.09$ & $* /-14.40$ & $* / 3.80-4.43$ \\
\hline$d=8 \mathrm{~mm}$ & $* / 4.02$ & $* /-13.71$ & $* / 3.80-4.42$ \\
\hline$d=10 \mathrm{~mm}$ & $* / 4.09$ & $* /-14.54$ & $* / 3.87-4.32$ \\
\hline$d=20 \mathrm{~mm}$ & $* / 3.98$ & $* /-16.59$ & $* / 3.70-4.32$ \\
\hline$d=30 \mathrm{~mm}$ & $3.86 / 3.95$ & $-32.48 /-$ & $3.70-4.27 /$ \\
& & 28.38 & $3.70-4.43$ \\
\hline Free-Space & $4 / 4.04$ & $-48.28 /-$ & $3.75-4.50 /$ \\
& & 28.93 & $3.75-4.55$ \\
\hline
\end{tabular}

\section{SIMULATED IN-BODY INVEST IGATIONS}

In this section, the in-body antenna performance is investigated by simulations.

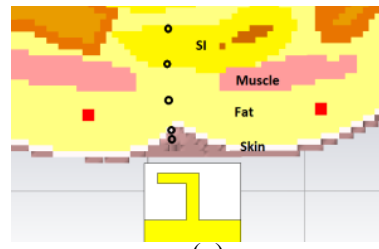

(a)

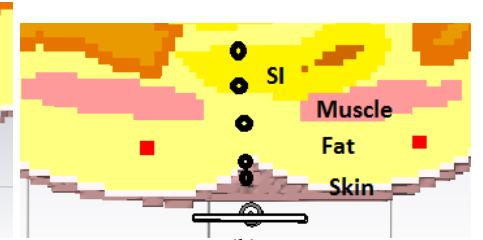

(b)
Fig. 8. Positions of the P-field probes at different tissue layers of the human body at $4 \mathrm{~mm}$ distance in (a) Perpendicular and (b) Parallel positions. The probe positions are marked with black cross and circle.

The antenna fulfillment for in-body communication, with a target placed at the SI tract, was evaluated in the rest of the paper using a realistic human body model. At this regard, five P-field (Power-field) probes were placed at distinct places inside the voxel model. The probe emplacement was chosen as follows: after the skin layer, middle of fat layer and just before SI layer. Moreover, because of the meandered shape of the SI tract, two points were taken into consideration: one at the middle of the first SI layer appearing in the line of sight of the antenna, and the other at the middle of the followed SI layer. These listed P-field probes were designed in Fig. 8 by five aligned cross and circle markers. The P-field results were calculated separately according to Fig. 8 (a) and Fig. 8 (b) for "Perpendicular" and "Parallel" antenna positions and detailed values were grouped in Table 2. In overall, it is clearly remarked that the power-field decreased in the P-probe order from skin to the second SI segment regardless the chosen antenna position. The resultant results show a power field value of $-2 \mathrm{~dB}$ and $7 \mathrm{~dB}$ after the skin and in a middle point of the fat layer for both antenna positions. By arriving at the first segment of the SI tract, the difference in power-field was distinguished. Higher value of $-12 \mathrm{~dB}$ was revealed at the interface of the first SI segment. However, by reaching the second SI segment, situated after the first one, the power field difference was minor of $-15 \mathrm{~dB}$ regardless the chosen antenna position. In conclusion, those results are favorable, gratifying the establishment of an in-body communication between the proposed antenna and a wireless capsule inside the SI tract, regardless the antenna position of the wearable external device (receiving antenna) by the patient. The latest conducted investigations proved that the antenna position, either "Parallel" or "Perpendicular", does not have any effect on the RF signal penetration of the SI layer. This again supports the idea that the "Parallel" position of the wearable on-body antenna is most likely better in practice for the WCE systems, since the antenna can be easily held and attached to the patient.

\section{CONCLUSION AND PERSPECTIVES}

The paper presents a compact UWB antenna operating at low UW B band of 3.75-4.25 GHz, defined in IEEE 802.15.6 standard for WBAN applications. The antenna has directional radiation pattern with high gain of $9 \mathrm{~dB}$ at $4 \mathrm{GHz}$. The antenna is developed to meet the requirements of an onbody antenna held by a patient under diagnostic for wireless capsule endoscopy. The diagnostic will be based on the improvement of the communication link between the proposed receiving antenna and a swallowed capsule antenna travelling the SI tract. Therefore, in this paper, the receiving antenna performances needed to be evaluated by taking into account the human body presence. That is why a voxel model was introduced to analyze the matching and the onbody effect of the proposed antenna. Besides the antenna was tested in "Parallel" and "Perpendicular" positions so that to extract the effect of the radiation shape on the body. Since the antenna should establish a wireless communication with the device (capsule) situated in the SI, the coverage of the antenna radiation of this body part was of prime focus. At this regard, the matching, radiation patterns and power-field probes were analyzed, confirming that the antenna radiation can reach the SI layer in both antenna positions "Parallel" and "Perpendicular". However, for this wireless capsule endoscopy application, "Parallel" antenna position is preferred for medical practical use. More investigations appealing the capsule presence will be delivered in a coming paper. For this end, a novel capsule design operating at the low-UWB frequency range will be described and an exhaustive communication link investigation with this proposed on-body antenna will be provided by evaluating the budget link of the communication at different body areas (stomach, small-intestine and large intestine) by using both antenna positions presented in this paper. Then the resulting simulation and measurement investigation results will be compared to available ones in literature to draw conclusions.

\section{ACKNOWLEDGEMENT}

This work was funded by the Academy of Finland 6Genes is Flagship, grant number 318927.

\section{REFERENCES}

[1] A. N. Hosoe; K. Takabayashi; H. Ogata and T. Kanai, "Capsule endoscopy for small-intestinal disorders: Current status", Digestive Endoscopy For Gastroenterologists and Endoscopic Surgeons, pp.?, 2019

[2] G. Shao ; Y. Tang; L. Tang; Q. Dai and Y-. X. Guo, "A Novel Passive Magnetic Localization Wearable System for Wireless Capsule Endoscopy", IEEE Sensors Journal, 2019.

[3] J-. J. Hao, L-. J. Lv, L. Jua, X. Xiea, Y-. J. Liua, H-. W. Yang Simulation of microwave propagation properties in human abdominal tissues on wireless capsule endoscopy by FDTD”, Biomedical Signal Processing and Control, Vol. 49, pp. 388-395, 2019.

[4] S. P-. Simbor; C. Andreu; C. G-. Pardo ; M. Frasson and N. Cardona, "UWB Path Loss Models for Ingestible Devices", IEEE Transactions on Antennas and Propagation, 2019. 
[5] D. Anzai ; K. Katsu ; R. C-. Santiago ; Q. Wang ; D. Plettemeier ; J. Wang and I. Balasingham, "Experimental Evaluation of Implant UWB-IR Transmission With Living Animal for Body Area Networks", IEEE Transactions on Microwave Theory and T echniques, Vol. 62,I. 1, pp. 183-192, 2014.

[6] T. Dissanayake ; K. P. Esselle and M. R. Yuce, "Dielectric Loaded Impedance Matching for Wideband Implanted Antennas", IEEE Transactions on Microwave Theory and Techniques, Vol. 57, I. 10, 2009.

[7] K. M. S. Thotahewa ; J-. M. Redoutè and M. R. Yuce, "Propagation, Power Absorption, and Temperature Analysis of UWB Wireless Capsule Endoscopy Devices Operating in the Human Body", IEEE Transactions on Microwave Theory and Techniques, Vol. 63, I. 11, 2015.

[8] T. Dissanayake ; M. R. Yuce and C. Ho, "Design and Evaluation of a Compact Antenna for Implant-to-Air UWB Communication", IEEE Antennas and Wireless Propagation Letters, Vol. 8, 2009.

[9] D. Anzai ; K. Katsu ; R. C-. Santiago ; Q. Wang; D. Plettemeier ; J. Wang and I. Balasingham, "Experimental Evaluation of Implant UWB-IR Transmission With Living Animal for Body Area Networks", Vol. 62, I. 1, 2014.

[10] Q. Wang; K. Wolf ; D. Plettemeier, “An UWB capsule endoscope antenna design for biomedical communications", 3rd International $\begin{array}{lll}\text { Symposium on Applied } & \text { Sciences }\end{array}$ in Biomedical and CommunicationTechnologies (ISABEL 2010), 2010 .
[11] IEEE Standard for Local and metropolitan area networks_Part 15.6: Wireless Body Area Networks, pp. IEEE Std 802.15.6-2012, pp. 1 271, 2012.

[12] C. Kissi; M. Särestöniemi; C. P-. Raez; M. Sonkki; and M. N. Srifi, "Low-UWB Directive Antenna for Wireless Capsule Endoscopy Localization", BODYNET S 2018: 13th EAI International Conference on Body Area Networks, pp.431-442, 2-3 October 2018, Oulu, Finland.

[13] C. Kissi ; M. Särestöniemi ; T. kumpuniemi ; M. Sonkki ; S. Myllymäki ; M. N. Srifi and C. P-. Raez, "On-body Cavity-backed Low-UWB Antenna for Capsule Localization”, International Journal of Wireless Information Networks (IJWIN), Vol. 27, pp. 30-44, 2020.

[14] C. Kissi ; M. Särestöniemi ; T. kumpuniemi ; M. Sonkki ; S. Myllymäki ; M. N. Srifi and C. P-. Raez, "Directive Low-Band UWB Antenna for In-body Medical Communications", IEEE Access, Vol. 7,pp. 149026-149038, 2019.

[15] C95.3-2002 - IEEE Recommended Practice for Measurements and Computations of Radio Frequency Electromagnetic Fields With Respect to Human Exposure to Such Fields, $100 \mathrm{kHz}-300 \mathrm{GHz}$, IEEE Std C95.3-2002 (Revision of IEEE Std C95.3-1991), pp. 1 - 126, 2003. 